\title{
SWebB: Semantic Web Browsing
}

\author{
Fausto Giunchiglia \\ Dept. of Information and Communication Technology \\ University of Trento, 38050 Povo, Trento, Italy \\ fausto@dit.unitn.it
}

In this talk I will present a browser, called $S W e b B$ (Semantic Web Browser), which explicitly uses, whenever available, the semantic information codified in Contextual Ontologies. Contextual Ontologies are ontologies enriched with Context Links. Ontologies define the conceptual model of a peer and describe its content. Context mappings are sets of bridge rules, namely pairs which allow to relate, via appropriate semantic relations (e.g., equivalence), concepts, roles and individuals in different ontologies. Using $S W e b B$, context mappings, similarly to standard Web links, can be discovered, navigated, copied, and so on. Contexts mappings are discovered by Semantic Matching. Contextual Ontologies are formalized using the C-OWL Language. The talk will also provide an overview of the key aspects of C-OWL.

\section{References}

1. F. Giunchiglia, P. Shvaiko. Semantic Matching. In The Knowledge Engineering Review Journal, 18(3) 2003.

2. L. Serafini, P. Bouquet, B. Magnini, S. Zanobini: Semantic Coordination: A new approach and an application. In proceedings of ISWC'03 (2003)

3. P. Bouquet, F. Giunchiglia, F. Van Harmelen, L. Serafini, H. Stuckenschmidt . C-OWL: contextualizing ontologies. "2nd international semantic web conference (ISWC 2003)", edited by Dieter Fensel and Katia p. Sycara and John Mylopoulos, Sanibel Island (Fla.), 20-23 October 2003, pp. 164-179

4. F. Giunchiglia, P. Shvaiko, M. Yatskevich. S-Match: An algorithm and an implementation of semantic matching. In Proceedings of ESWS'04, pages 61-75, 2004.

5. F. Giunchiglia, M. Yatskevich, E. Giunchiglia. Efficient Semantic Matching. In Proceedings of ESWC'05, 2005. 